\title{
Intestinal tumours, colonic butyrate and sleep in exercised Min mice
}

\author{
Laura Basterfield $^{1 *}$ and John C. Mathers ${ }^{2}$ \\ ${ }^{1}$ Faculty of Medical Sciences, Human Nutrition Research Centre, Institute of Health and Society, Newcastle University, Newcastle \\ upon Tyne NE2 $4 H H, U K$ \\ ${ }^{2}$ Faculty of Medical Sciences, Human Nutrition Research Centre, Institute for Ageing and Health, Newcastle University, Newcastle \\ upon Tyne NE2 $4 H H$, $U K$
}

(Received 5 October 2009 - Revised 26 January 2010 - Accepted 28 January 2010 - First published online 25 March 2010)

There is strong epidemiological evidence that more physical activity is associated with reduced risk of colon cancer, but the amount or type of activity necessary to invoke this protection is disputed, and the mechanism that is responsible has not been elucidated. The present study compared the effects of two contrasting exercise regimens on intestinal tumourigenesis in Min mice, and investigated two novel mechanistic factors: colonic butyrate and sleep. From 5 weeks of age, Min mice were exercised by running on a treadmill (TR; $\leq 21 \mathrm{~m} / \mathrm{min}, 30-60 \mathrm{~min} / \mathrm{d}, 5 \mathrm{~d} / \mathrm{week}, \leq 12$ weeks). Additional groups of mice were provided with an exercise wheel (WH) or no exercise (CON). Mice had free access to a Westernstyle, high-fat diet. WH mice ran $3.97 \mathrm{~km}$ (females) and $1.92 \mathrm{~km}$ (males) daily $(P=0.002)$. There were no differences in body weight gain or body composition between treatment groups. Treadmill running reduced the numbers of larger $(\geq 2 \mathrm{~mm}$ diameter $)$ tumours $(P=0 \cdot 042)$, and tended to reduce tumour multiplicity in the colon $(P=0.049)$. TR mice had a higher molar proportion of butyrate in colonic digesta than CON mice $(P=0.030)$, and when treatment groups were combined, there was a weak negative correlation $(r-0 \cdot 174, P=0 \cdot 061)$ between butyrate molar proportion and total tumour number. In a subset of animals in which non-exercise physical activity was monitored, there were strong positive correlations between sleep duration and both tumour multiplicity $(P<0.001)$ and tumour burden $(P=0 \cdot 001)$. More studies of the effects of sleep and of colonic butyrate in mediating the effects of physical activity on intestinal tumourigenesis are warranted.

Min mice: Exercise: Butyrate: Sleep: Tumours

There is substantial epidemiological evidence (at least fifty studies $^{(1)}$ ) to suggest that being physically active can reduce the risk of developing cancer, particularly of the breast and colon $^{(2)}$. Indeed, it has been estimated that high levels of physical activity may reduce the risk of colon cancer by up to $50 \%{ }^{(3)}$. However, physical activity may not help those with a family history of colonic adenomatous polyps ${ }^{(4)}$ or those who have had at least one polyp removed, as physical activity had no effect on polyp recurrence in the Polyp Prevention Trial ${ }^{(5)}$. There may be sex differences in response to physical activity since there was an inverse association between physical activity and polyp recurrence in men only in the Calcium Polyp Prevention Study ${ }^{(6)}$.

A limitation of such observational studies is that they demonstrate associations, but cannot prove causality, between physical activity and colon cancer, and there may be other aspects of lifestyle associated with physical activity (such as diet ${ }^{(7)}$ ) that are responsible for the protection against colon cancer. In addition, because physical activity is a complex and heterogeneous behaviour, it is difficult to characterise and quantify, and this imprecision in measurement of exposure exacerbates the difficulty in assessing observational data.

The amount or types of physical activity which may reduce risk of bowel cancer are poorly understood. Human epidemiological studies have used relatively crude instruments to quantify physical activity, including duration and intensity of leisure activity, occupational activity, activity in the past year and lifetime activity. Evidence of the benefit (or otherwise) of specific forms of exercise is obtained more readily from experiments in animal models (as reviewed by Basterfield et al. ${ }^{(8)}$ ). Studies using carcinogen-treated rats have been unanimous in finding reduced colon cancer with both voluntary (wheel running) and forced (treadmill running) exercise ${ }^{(9-13)}$. In contrast, the outcomes of studies of exercise in Min mice, which carry a truncating mutation in the $A p c$ gene and develop multiple intestinal tumours spontaneously ${ }^{(14)}$, have been mixed with only a few studies showing protection against tumourigenesis in some circumstances ${ }^{(15-20)}$. The reasons for this heterogeneity in response are not known, but may be related to the use of different sexes, exercise protocols and/or diets. The type of physical activity (treadmill or wheel running), and how much, that protects against intestinal tumourigenesis in Min mice remains unclear, and the mechanisms responsible for protection remain to be discovered.

Poor sleep quality is associated with the metabolic syndrome $^{(21)}$ and obesity ${ }^{(22)}$, and obesity is known to be a risk factor for cancer ${ }^{(7)}$. Interest in the association between

Abbreviations: CON, control treatment; TR, treadmill treatment; WH, wheel treatment.

* Corresponding author: Dr Laura Basterfield, fax +44 191222 5581, email laura.basterfield@ncl.ac.uk 
sleep and cancer is developing ${ }^{(23)}$, although results so far are conflicting ${ }^{(24-28)}$. To date, the relationship between sleep and intestinal cancer risk has not been investigated using an animal model of tumourigenesis. The SCFA butyrate is another potentially protective factor which is underinvestigated in exercise studies. SCFA are produced by the bacterial fermentation of carbohydrates in the large intestine, and butyrate is used as fuel by colonocytes ${ }^{(29)}$. The antiinflammatory and anti-neoplastic properties of butyrate are well established $^{(30)}$, but its potential role as a mediator of the effects of physical activity has not been investigated.

The primary purpose of the present study was to compare the effects of forced (treadmill running) and voluntary (wheel running) modes of exercise on tumourigenesis in Min mice. To maximise the potential for translation of the study outcomes to human health, the mice were fed a Western-style, high-fat diet. We hypothesised that mice on a wheel running exercise protocol would undertake a greater amount of physical activity than their treadmill-running counterparts, and so would have fewer, and smaller, tumours than either treadmill-running or unexercised control mice. Furthermore, we explored two novel protective mechanisms, namely increased butyrate proportion in the large bowel and, in a subset of mice, quantity of non-exercise physical activity and sleep.

\section{Experimental methods}

\section{Animals}

All procedures complied with current UK government Home Office legislation, and were approved by the Newcastle University Ethics Committee. Female C57BL/6 mice (Harlan, Oxon, UK) were mated with male C57BL/6Min + / - mice (Min mice) from the established Newcastle University colony, and offspring were genotyped at 4 weeks of age using DNA from tail snips according to established methods ${ }^{(31)}$. For three separate studies, 155 Min mice (eighty-seven females and sixty-eight males) were available and randomised to one of three treatment groups at approximately 5 weeks of age: treadmill (TR) thirty-one females and twenty-nine males, wheel (WH) twenty-six females and seventeen males, or control (CON) thirty females and twenty-two males. TR mice were housed $1-6$ in a cage, and were allowed to run on a treadmill (Exer-3/6 rodent treadmill, Columbus Instruments, $\mathrm{OH}, \mathrm{USA}$ ) for $5 \mathrm{~d} /$ week at $18-21 \mathrm{~m} / \mathrm{min}$ for $30-60 \mathrm{~min}$ on a $5 \%$ gradient for $10-12$ weeks. The first 2 weeks were used to train the mice to run on the treadmill, and during this period, the speed and duration of running were increased gradually. WH mice were housed individually in a cage containing a running wheel to which they had free access. The running wheel had an internal diameter of $10 \mathrm{~cm}$ for the first 5 weeks and then changed to a $13 \mathrm{~cm}$ internal diameter wheel for the final 5 weeks. Numbers of wheel revolutions were recorded by a magnetically actuated counter. CON mice were housed $1-6$ in a cage, and were removed to individual 'control lanes' (no imposed exercise), while the TR mice were on the treadmill. All mice were housed in the same room as the treadmill under a $12 \mathrm{~h}$ light-dark cycle, and were offered $6 \mathrm{~g} / \mathrm{d}$ of a non-commercial 'Western'-style ${ }^{(32)}$, high-fat diet containing $(\mathrm{g} / \mathrm{kg})$ casein high nitrogen 279.49 , lard 250, maize flour 250, sucrose 148, mineral mix AIN-93-G-MX 35, gelatine 20, vitamin mix AIN-93-VX 10, L-cystine 3 , choline bitartrate $2 \cdot 5, \mathrm{Cr}_{2} \mathrm{O}_{3} 2$ and $t$-butylhydroquinone $0 \cdot 014$. Due to its high fat content, this diet had a 'paste-like' consistency, which minimised scattering by the mice and facilitated the collection of uneaten food. Food refusals were weighed daily. Body weights were recorded at the beginning of each week.

\section{Non-exercise physical activity and quantification of sleep}

An IR-sensing device ('Inframot', TSE Systems, Bad Homburg, Germany) was used to quantify cage activity in a subsample of singly housed mice, seven TR (five males and two females) and five CON (three males and two females). Inframot detects movements of $5 \mathrm{~ms}$ length and greater, and stores the data accumulated in set time periods as 'counts'. Each mouse was assessed for $23 \mathrm{~h}$ (i.e. when not on the treadmill/control lanes) to provide information on the total amount of activity and the activity during the light and dark phases. Activity was accumulated in $10 \mathrm{~min}$ bouts. Time spent sleeping was determined according to the strict criterion of zero movement counts recorded in each $10 \mathrm{~min}$ recording epoch ${ }^{(33)}$.

\section{Blood and tissue sampling}

Mice were killed after 10-12 weeks of treatment. Anaesthesia was induced using $3 \%$ isoflurane in oxygen followed by cardiac exsanguination and cervical dislocation. Blood was collected into EDTA for haematocrit measurement. The carcass was weighed, and a midline section was made. The intestinal organs and spleen were dissected out, weighed and examined for the presence of tumours by an observer blind to the treatment group. The small intestine was divided into equal-length proximal and distal portions and opened longitudinally. Size and site of all adenomas were recorded. The colon contents were mixed with de-proteinising solution (containing $100 \mathrm{~g} / 350 \mathrm{ml} \mathrm{H}_{2} \mathrm{O}$ metaphosphoric acid and $50 \mathrm{~mm}$ 3-methyl valeric $\operatorname{acid}^{(34)}$ ) in preparation for SCFA determination by GC. Carcasses were freeze-dried, and body fat was measured gravimetrically after extraction by petroleum diethyl ether using the Soxhlet procedure.

\section{Statistical methods}

Data were tested for normality, and any data that were not normally distributed were transformed. Normally distributed and transformed data were investigated for effect of exercise using the general linear model of ANOVA, with sex included as a covariate. Comparisons between treatment groups (TR $v$. $\mathrm{CON}$ and $\mathrm{WH} v$. CON) were investigated using Dunnett's test. As data from three replicate studies were combined in this analysis, 'study' was included in the analysis as a fixed factor. Normally distributed data are presented as mean values with their standard errors. The results for transformed data are summarised as geometric means and $95 \% \mathrm{CI}$. The data for colon tumours and small tumours $(\leq 1 \mathrm{~mm}$ diameter) were tested using the non-parametric Kruskal-Wallis test, and are presented as medians and ranges. The significance level was set at $P \leq 0 \cdot 05$. 
Table 1. Food intake, weight gain and percentage body fat of mice by exercise group (Mean values with their standard errors and $95 \%$ confidence intervals)

\begin{tabular}{|c|c|c|c|c|c|c|c|c|c|c|c|}
\hline \multirow[b]{2}{*}{ Variable } & \multicolumn{3}{|c|}{$\operatorname{TR}(n 41)$} & \multicolumn{3}{|c|}{$\mathrm{WH}(n 43)$} & \multicolumn{3}{|c|}{$\operatorname{CON}(n 52)$} & \multicolumn{2}{|c|}{ Probability } \\
\hline & Mean & SEM & $95 \% \mathrm{Cl}$ & Mean & SEM & $95 \% \mathrm{Cl}$ & Mean & SEM & $95 \% \mathrm{Cl}$ & TR v. CON & $\mathrm{WH} v \cdot \mathrm{CON}$ \\
\hline Food intake $(\mathrm{g} / \mathrm{d})$ & 2.08 & 0.03 & & $2 \cdot 26$ & 0.03 & & $2 \cdot 14$ & 0.03 & & 0.364 & 0.018 \\
\hline Weight gain over study (g) & $7 \cdot 3$ & & $6 \cdot 3,8 \cdot 4$ & $7 \cdot 2$ & & $6 \cdot 2,8 \cdot 3$ & $8 \cdot 1$ & & $7 \cdot 1,9 \cdot 1$ & 0.459 & 0.399 \\
\hline Body fat (\%) & 30.9 & 2.09 & & 23.5 & 1.82 & & 23.9 & 2.05 & & 0.037 & 0.979 \\
\hline
\end{tabular}

TR, treadmill running; WH, wheel running; CON, control (not exercised).

\section{Results}

Seven female mice and twelve male mice did not complete the initial 2-week training period on the treadmill satisfactorily, and were removed from the study, as per previous investigations $^{(16,17)}$, leaving twenty-four female and seventeen male mice in the TR treatment group. A higher proportion of female $(18 / 24)$ than of male $(8 / 17)$ mice ran on every possible day $(P=0 \cdot 048)$. There was no difference between the sexes in the median time spent on the treadmill in each session. As they completed the majority of the exercise protocol (twenty-six mice ran on all days, nine ran between 81 and $99 \%$ of days, and six ran between 68 and $80 \%$ of days), results from all these mice are included in this analysis (intention to treat analysis). During the 10-week study, female WH mice ran approximately twice as far $(P=0.002)$ in their wheels as did males, and the mean distances run were $276($ SEM 26.0) v. 136 (SEM 32.1) km, which equate to 3.97 and $1.92 \mathrm{~km} / \mathrm{d}$ for female and male mice, respectively.

WH mice consumed significantly $(P=0.015)$ more food than $\mathrm{CON}$ mice (Table 1) whose intake was similar to that of TR mice. There was no difference between treatment groups for either carcass weight, weight gained over the study or body composition (Table 1). Weekly weight gain was similar for each treatment group, except at week 6 when CON mice gained $0.5 \mathrm{~g}$ more than both TR and $\mathrm{WH}$ mice $(P=0.017$ for TR $v$. CON, $P=0.021 \mathrm{WH} v$. CON).

Total numbers of intestinal tumours were very similar for WH, CON and TR mice (Table 2), but TR mice had significantly $(P=0.042)$ fewer larger $(\geq 2 \mathrm{~mm})$ tumours than CON mice. TR mice showed a trend for fewer tumours in all sections of the intestine, but, with the exception of the colon $(P=0 \cdot 049)$, the differences between TR and CON mice were NS $(P>0.05)$ when analysed by anatomical site. For the WH mice, there was no correlation between distance run in wheels in 10 weeks and the total number of tumours ( $r-0.096, P=0.539$ ), or the tumour burden (the sum of tumour diameters, $r-0 \cdot 120, P=0.444)$. Combined data showed a negative correlation between weight gained over the study and total tumour number $(r-0 \cdot 372, P<0 \cdot 001)$.

The haematocrits of TR (29.6 (SEM 1.32)\%) and WH (28.4 (SEM 1.49) \%) mice were very similar, and both were significantly higher than that of CON (24.5 (SEM 1.28)\%) mice $(P=0.009$ and 0.040 , respectively). The spleens of the two exercising groups were significantly lighter (TR $197 \mathrm{mg}$, WH $247 \mathrm{mg}$ ) than those of the CON mice $(334 \mathrm{mg}$; $P=0.007$ and 0.055 for comparisons of TR and WH with $\mathrm{CON}$, respectively). There was a strong inverse correlation $(r-0.580, P<0.001)$ between spleen weight and haematocrit (Fig. 1), and spleen weight was positively correlated with tumour number $(r 0 \cdot 373, P=0 \cdot 002)$.

The type of exercise had a significant effect on the pattern of colonic SCFA (Table 3). For TR mice, the reduction in acetate molar proportion approached significance compared with the CON mice $(P=0.067)$, whereas butyrate molar proportion was increased by $50 \%$ compared with the CON mice $(P=0.030)$. For WH mice, propionate was increased significantly $(P=0.019)$ compared with the $\mathrm{CON}$ mice. This observation of differential effects of exercise regimen on colonic SCFA pattern highlights an area of ignorance about the impact of physical activity on intestinal function. It may be relevant to note that treadmill running was restricted to

Table 2. Tumour numbers and anatomical distribution of tumours in Min mice by exercise group

(Mean values and $95 \%$ confidence intervals)

\begin{tabular}{|c|c|c|c|c|c|c|c|c|}
\hline \multirow[b]{2}{*}{ Variable } & \multicolumn{2}{|c|}{$\operatorname{TR}(n 41)$} & \multicolumn{2}{|c|}{$\mathrm{WH}(n$ 43) } & \multicolumn{2}{|c|}{$\operatorname{CON}(n 52)$} & \multicolumn{2}{|c|}{ Probability } \\
\hline & Mean & $95 \% \mathrm{Cl}$ & Mean & $95 \% \mathrm{Cl}$ & Mean & $95 \% \mathrm{Cl}$ & TR $v$. CON & WH v. CON \\
\hline Total tumours & $11 \cdot 0$ & $9 \cdot 1,13 \cdot 2$ & $13 \cdot 9$ & $11 \cdot 3,17 \cdot 1$ & $14 \cdot 1$ & $11 \cdot 8,16 \cdot 8$ & 0.084 & 0.993 \\
\hline Proximal SI tumours & $5 \cdot 1$ & $4 \cdot 2,6 \cdot 1$ & $6 \cdot 0$ & $4 \cdot 8,7 \cdot 4$ & 7.5 & $6 \cdot 0,9 \cdot 5$ & 0.855 & 0.612 \\
\hline Distal SI tumours & 5.5 & $4 \cdot 3,7 \cdot 1$ & $6 \cdot 4$ & $4.9,8.4$ & 7.5 & $6 \cdot 0,9 \cdot 5$ & 0.111 & 0.479 \\
\hline Total SI tumours & $10 \cdot 7$ & $8.9,12.8$ & $13 \cdot 4$ & $10 \cdot 8,16 \cdot 5$ & 13.4 & $11 \cdot 2,16 \cdot 1$ & 0.125 & 0.998 \\
\hline Colon tumours ${ }^{\star}$ & 0.0 & $0.0-3 \cdot 0$ & 0.0 & $0.0-5 \cdot 0$ & 1.0 & $0.0-4.0$ & 0.049 & 0.126 \\
\hline Small tumours $(\leq 1 \mathrm{~mm})^{\star}$ & 1.0 & $0.0-32.0$ & 0.0 & $0.0-31.0$ & 1.0 & $0.0-17 \cdot 0$ & 0.617 & 0.622 \\
\hline Large tumours ( $\geq 2 \mathrm{~mm}$ ) & $10 \cdot 1$ & $9 \cdot 3,11 \cdot 0$ & $12 \cdot 7$ & $11 \cdot 7,13 \cdot 8$ & $13 \cdot 1$ & $12 \cdot 1,14 \cdot 1$ & 0.042 & 0.953 \\
\hline Mean tumour diameter (mm) & $2 \cdot 6$ & $2 \cdot 5,2 \cdot 7$ & $2 \cdot 4$ & $2 \cdot 3,2 \cdot 5$ & 2.5 & $2 \cdot 4,2 \cdot 5$ & 0.300 & 0.899 \\
\hline Tumour burden $(\mathrm{mm}) \dagger$ & $29 \cdot 3$ & $24 \cdot 8,34 \cdot 7$ & $34 \cdot 3$ & $28 \cdot 3,41 \cdot 5$ & 35.4 & $30 \cdot 0,41 \cdot 8$ & 0.183 & 0.944 \\
\hline
\end{tabular}

TR, treadmill running; WH, wheel running; CON, control (not exercised); SI, small intestine.

${ }^{*}$ Median and range.

† Sum of all tumour diameters. 


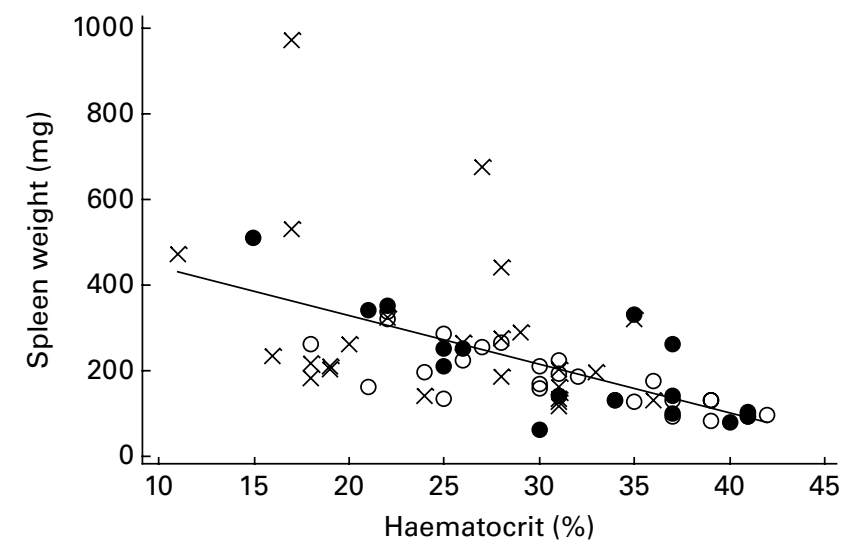

Fig. 1. Negative correlation $(P<0.001)$ between spleen weight and haematocrit in Min mice. Spleen weight $=554$ (SE 58) -11 (SE 2) $\times$ haematocrit. •, Treadmill running (TR) mice; $\bigcirc$ wheel $(\mathrm{WH})$ running mice, $\times$, control (CON) or no exercise mice.

the light phase of the day, whereas the majority of the wheel running occurred in the dark phase. Given the diurnal patterns of food consumption by mice that were fed ad libitum (most food is eaten in the dark phase of the day), there may be some interaction between timing of food ingestion, exercise and large bowel bacterial activity. When the treatment groups were combined, there was a negative correlation ( $r-0.174, P=0.061)$ between butyrate molar proportion and total tumour number.

Non-exercise physical activity was measured in a subsample of twelve mice (seven TR and five $\mathrm{CON}$ ), with a mean of five separate $23 \mathrm{~h}$ continuous recordings per mouse. With this subsample of mice, there was no significant $(P>0.05)$ difference in tumour number or tumour burden between the groups, but the TR mice tended to have fewer tumours (12.1 (SEM 4.3) for TR and 24.1 (SEM 5.0) for CON, $P=0 \cdot 104)$ in parallel with the findings of the main study. During the $23 \mathrm{~h}$ per day when they were in their home cage, there was a trend for TR mice to be more active than CON mice $\left(1377 v .757 \times 10^{3}\right.$ counts, $\left.P=0.084\right)$, with this difference in activity being confined to the dark period. There was no difference in total sleep time between the groups $(P=0.125)$, or in sleep during the dark $(P=0.111)$ or light $(P=0.793)$ phase. However, there were negative correlations between total activity and tumour burden (Pearson's $r-0.559, P=0.059$, Fig. 2(a)), total activity and tumour number $(r-0.558, P=0.059$, Fig. 2(b)), and dark activity and tumour burden $(r-0 \cdot 559, P=0 \cdot 040)$. There were significant positive correlations between dark sleep and tumour burden ( $r$ 0.835, $P=0.001$, Fig. 2(c)), total sleep time and tumour number $(r 0.872, P<0.001$, Fig. 2(d)), and total sleep and tumour burden $(r 0.841, P=0.001)$. There was no correlation between activity and final body mass.

\section{Discussion}

The present study adds to the body of work on the effects of exercise on tumourigenesis in Min mice by demonstrating a small reduction in larger tumours $(P=0.042)$ and in colon tumours $(P=0.049)$ following treadmill running. We report the novel observation of altered SCFA proportions in the colon, in particular, an increase in the anti-neoplastic butyrate, in response to treadmill running. Furthermore, we provide preliminary evidence that time spent sleeping is correlated strongly with both tumour number and tumour burden.

The aim of the present study was to help resolve controversies arising from the six previously published studies of the effect of exercise on tumourigenesis in Min mice, which have reported conflicting results (see Table 4 for summary). In contrast with the heterogeneity of outcomes from Min mouse studies, there were consistently protective effects of both voluntary and involuntary forms of exercise in previous experiments with carcinogen-treated rats ${ }^{(9-13)}$. There is no readily apparent reason for the inconsistent effects of exercise in the Min mouse studies, but differences in experimental protocols between the studies, e.g. the ages at the start of the procedures, the length of the study and the number of tumours generated per mouse, may help explain the differing results (Table 4). In the rat studies where a protective effect of exercise was apparent, only males were used, whereas both male and female Min mice have been used in most studies. There may be interactions between sex and environmental factors on tumourigenesis in Min mice since McKay et al. ${ }^{(35)}$ observed more tumours in females than in males, and this difference and total tumour numbers were reduced by feeding a low-folate diet.

The optimum mode of exercise (forced treadmill running or voluntary wheel running) to reduce tumourigenesis in Min mice has not been established yet. Although it seems counter-intuitive, treadmill running (which results in shorter running distances than wheel running) reduced tumour

Table 3. Effect of treadmill (TR) and wheel (WH) running on molar proportions ( $\mathrm{mmol} / \mathrm{mol})$ of SCFA in the colon of Min mice compared with the control (CON) animals

(Mean values and $95 \%$ confidence intervals)

\begin{tabular}{|c|c|c|c|c|c|c|c|c|}
\hline \multirow[b]{2}{*}{ SCFA } & \multicolumn{2}{|c|}{$\operatorname{TR}(n 30)$} & \multicolumn{2}{|c|}{ WH $(n 47)$} & \multicolumn{2}{|c|}{$\operatorname{CON}(n 41)$} & \multicolumn{2}{|c|}{ Probability of effect } \\
\hline & Mean & $95 \% \mathrm{Cl}$ & Mean & $95 \% \mathrm{Cl}$ & Mean & $95 \% \mathrm{Cl}$ & TR $v . \mathrm{CON}$ & WH v. CON \\
\hline Acetate & 748 & 723,773 & 757 & 737,777 & 782 & 758,807 & 0.067 & 0.152 \\
\hline Propionate & 107 & 95,120 & 123 & 112,135 & 103 & 92,115 & 0.842 & 0.019 \\
\hline Isobutyrate & 14 & 12,16 & 16 & 14,17 & 14 & 12,16 & 0.942 & 0.243 \\
\hline Butyrate & 75 & 58,95 & 50 & 39,62 & 50 & 38,62 & 0.030 & 0.999 \\
\hline Isovalerate & 20 & 17,22 & 21 & 19,23 & 19 & 17,21 & 0.717 & 0.129 \\
\hline Valerate & 13 & 9,16 & 12 & 10,14 & 10 & 7,13 & 0.327 & 0.430 \\
\hline
\end{tabular}

TR, treadmill running; $\mathrm{WH}$, wheel running; CON, control (not exercised). 
(a)

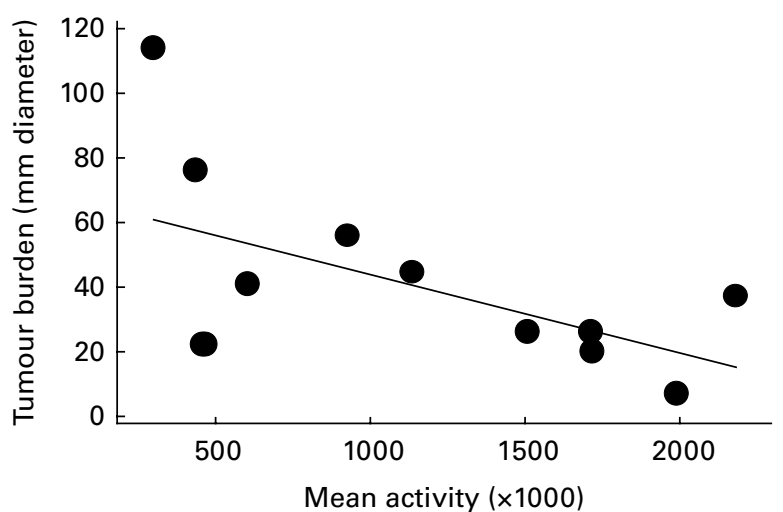

(c)

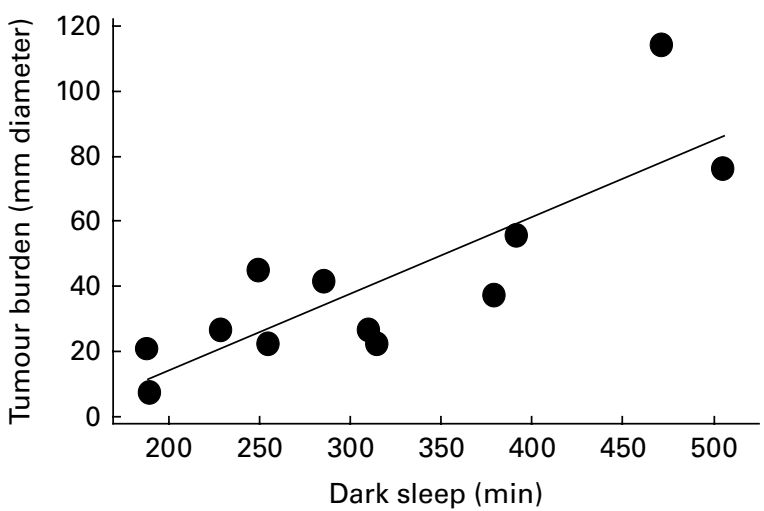

(b)

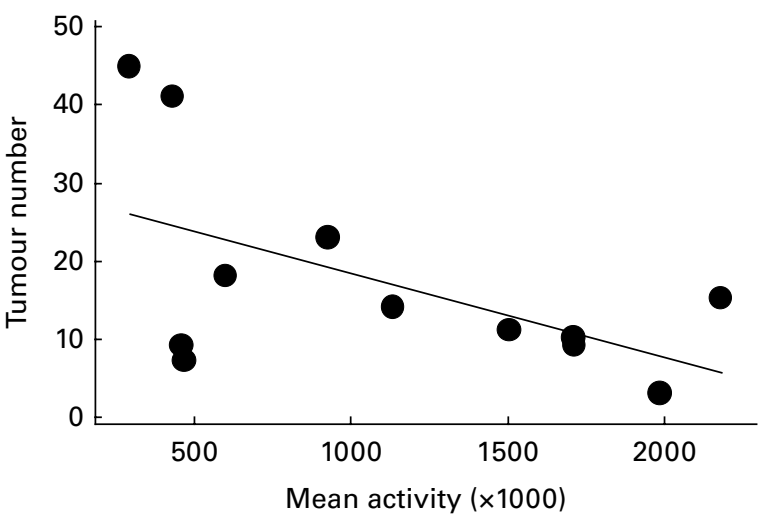

(d)

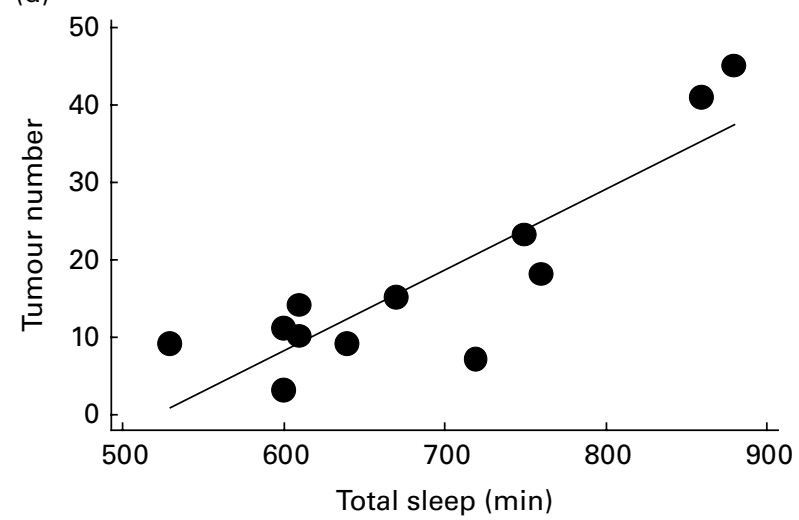

Fig. 2. Relationships between $23 \mathrm{~h}$ activity and (a) tumour burden $(r-0.559, P=0.059)$ and (b) tumour number $(r-0.558, P=0.059)$, and between (c) dark sleep (sleep during the dark period) and tumour burden $(r 0.835, P=0.001)$ and $(d)$ total sleep and tumour number $(r 0.872, P<0.001)$ in a subsample of Min mice. Symbols refer to individual mice.

number in male Min mice, whereas wheel running had no effect in the study done by Mehl et al. ${ }^{(17)}$. This mode of exercise difference was confirmed in the present study where tumours were smaller and numbers were lower in all sections of the intestine of TR mice but not of WH mice (Table 2).

Increased adiposity is a risk factor for cancer $^{(7)}$, and energetic restriction studies (which reduce body mass and adiposity) decrease tumour risk in human subjects ${ }^{(36)}$ and Min mice ${ }^{(37)}$. Therefore, if exercise lowers body mass it may also lead to a reduction in tumour load. Four of five carcinogen-treated rat studies $^{(9-12)}$ reported that exercise decreased body weight, and a similar effect was observed in two Min mice studies ${ }^{(16,18)}$ (one of which found an inverse relationship between polyp number and percentage body $\mathrm{fat}^{(18)}$ ), but not in the other two studies, neither of which reports any associations between body weight and tumour multiplicity ${ }^{(15,17)}$. Ju et al. ${ }^{(19)}$ reported a reduction in body fat but not in body mass with wheel running exercise in female Min mice, and Baltgalvis et al. ${ }^{(20)}$ did not observe a reduction in either body mass or fat pad mass with exercise. In the present study, we did not observe an effect of exercise on either final weight or body composition, in agreement with recent studies reporting no effect of wheel running on body mass or fat mass in female C57BL/6 mice ${ }^{(38)}$. However, where adiposity is measured at the end of the studies of tumourigenesis in Min mice (i.e. all studies to date), outcomes should be interpreted with caution since cachexia accompanying intestinal tumourigenesis may have profound effects on food intake and body composition. Colbert et al. ${ }^{(18)}$ observed that male wheel-running mice had higher levels of body fat, and suggested that this may indicate that WH mice were healthier than the CON mice which showed signs of cachexia (loss of muscle and fat tissue) ${ }^{(18,39)}$. A recent study ${ }^{(40)}$ has suggested that the severest cachectic symptoms are observed in Min mice with the highest levels of circulating IL-6 and greatest tumour multiplicity. Although we did not find a significant correlation between tumour number and body fat $(r-0.214, P=0.098)$, an observation also noted by $\mathrm{Ju}$ et al. ${ }^{(19)}$ and Baltgalvis et al. ${ }^{(20)}$, there was a significant negative correlation between total tumour number and weight gained over the study $(P<0 \cdot 001)$.

Inter-study differences in tumour outcome with different modes of exercise may be explained by differential effects on the energy balance of the experimental mice. Most previous studies of effects of exercise in Min mice have used low-fat diets, but tumour number can be increased by feeding a higher fat diet ${ }^{(41)}$. Our mice were given a high-fat diet that reflected human Western diets, as high-fat diets are associated with increased risk of colon cancer in human subjects ${ }^{(42)}$. WH mice, but not TR mice, ate significantly more than CON mice, presumably to compensate for their extra energy expenditure. This greater energy intake by the WH mice may explain the lack of difference in body weights compared with CON mice. The provision of energy-dense food ad libitum in the 
WS British Journal of Nutrition

Table 4. Comparison of published studies of the effects of exercise on intestinal neoplasia in Min mice*

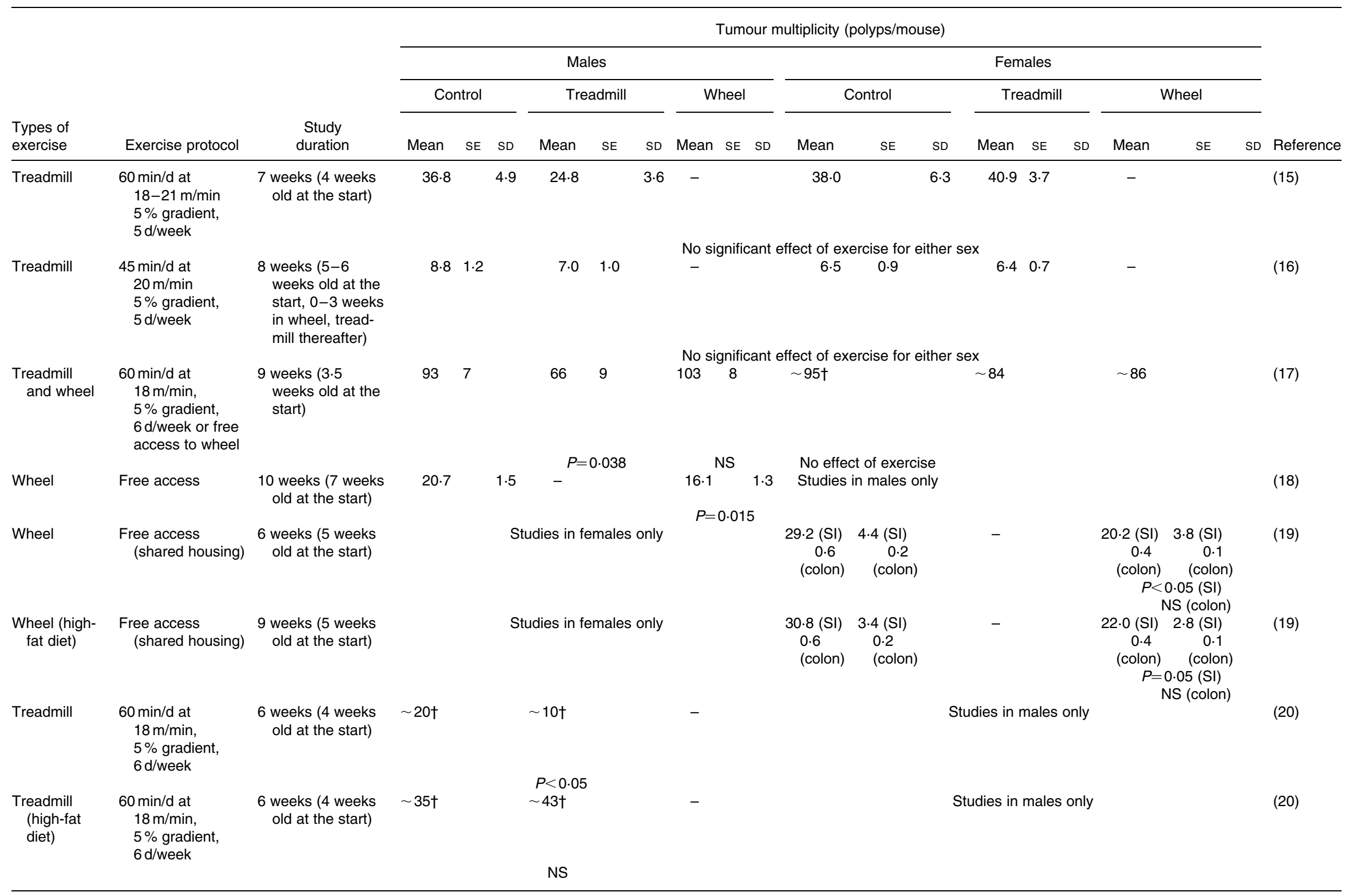

*Adapted from Basterfield et al. ${ }^{(8)}$

† Exact data not published. 
present study may have enabled our exercising mice to match their greater energy outputs with higher energy intakes, so preventing any change in energy balance, and this may explain the more modest effects of exercise on tumourigenesis seen in the present study. In contrast, some previous studies have 'pair-matched' food intake of exercising mice with that of non-exercising controls to induce negative energy balance $^{(16,18)}$. Mehl et al. ${ }^{(17)}$ who fed their mice ad libitum also found reduced tumour numbers with only treadmill running, and reported increased food intake by WH-running mice. Baltgalvis et al. ${ }^{(20)}$ showed that male TR mice on a low-fat diet had fewer tumours than controls, an effect that was negated by feeding a high-fat diet ${ }^{(20)}$. Thus, changes in energy balance may be more important than energy intake per se in determining effects on intestinal tumourigenesis, and this area deserves further exploration.

Insulin-like growth factor 1 (IGF-1) ${ }^{(16,18)}$, IL- $6^{(17)}$, corticosterone $^{(16,18)}$, inflammation ${ }^{(17,20)}$ and leptin ${ }^{(16,20)}$ have been investigated as possible mechanisms or mediators for the protective effect of exercise, but with no clear results. In the present study, we investigated the possibility that exercise alters the exposure of intestinal epithelial cells (the cells from which tumours develop) to pro- or anti-neoplastic substrates. We focused on butyrate which is one of the most potent anti-neoplastic components of the intestinal milieu and which suppresses tumour cell proliferation, increases apoptosis, induces differentiation and enhances immunosurveillance $^{(30)}$. Although several factors in food are known to alter SCFA concentrations ${ }^{(34,43)}$ and changes in the microbiota in the large intestine in response to diet are associated with reduced tumour number in Min mice ${ }^{(44)}$, the present study is the first to report effects of exercise on colonic SCFA in Min mice. We observed that the colon contents of TR mice contained a significantly $(P=0.030)$ greater proportion of butyrate than those of $\mathrm{CON}$ mice, and this greater exposure to butyrate may help to explain the reduced colonic tumour number and smaller tumours in TR mice. There was a negative correlation $(r-0.174, P=0.061)$ between butyrate molar proportion and total tumour number in the present study. The mechanism through which exercise alters intestinal production of butyrate (and other SCFA) is not known, but evidence from rats indicates that changes in the caecal microbiota may be critical in mediating increases in caecal butyrate $^{(45)}$. There are several potential means by which exercise could affect intestinal microbiota, including changes in intestinal transit time ${ }^{(46)}$, intestinal immune system or endogenous substances such as mucin ${ }^{(45)}$. In the present study, we found no effect of exercise on caecal transit time (data not shown). However, these observations suggest that future intervention studies should also investigate butyrate production.

There is increasing interest in the role of sleep in modulating cancer risk with evidence that increased sleep may reduce the risk of both breast ${ }^{(24,25,27)}$ and prostate cancers ${ }^{(26)}$. In contrast, other studies have reported no convincing evidence for a relationship between sleep duration and breast cancer risk $^{(28)}$ or a modest increased risk with greater sleep duration $^{(47)}$. Relationships between sleep and bowel cancer risk are not known. We observed strong positive correlations between sleep duration and tumour multiplicity $(P<0 \cdot 001)$ and sleep during the dark phase and tumour burden
$(P=0.001$; Fig. 2). It has been hypothesised that there is mutual reinforcement between circadian rhythms of melatonin production, the sleep/wake cycle and immune function ${ }^{(23)}$ and evidence that biological clock proteins may interact with the cell cycle/DNA damage response pathway to alter cancer risk. Others ${ }^{(48)}$ have observed an increase in sleep with increased body weight in mice fed a high-fat diet, and the complex interrelationships between physical activity, sleep, energy balance and cancer risk are a potentially important new research focus. The availability of robust techniques for quantifying sleep duration in animal models such as Min mice provides an exciting opportunity to investigate the putative relationship between sleep patterns and development of cancer.

Stress is another potentially important mediator of the effects of exercise regimens on intestinal tumourigenesis. TR mice were potentially exposed to extra stress during the current regimen, as the treadmill protocol occurred during the light phase of the day (when mice are often asleep), whereas most WH activity took place during the dark phase. Our investigation of non-exercise physical activity, however, suggests that TR mice did not behave differently from $\mathrm{CON}$ mice after they were returned to their home cages, as the amount of activity and sleep during the light period (when the intervention occurred) was not different between the two groups. This suggests that treadmill running is no more stressful than moving the $\mathrm{CON}$ mice to the individual control lanes, but the effect of exercise in the light phase $v$. dark phase of the day on intestinal tumourigenesis remains to be investigated. Our observation that greater non-exercise physical activity was associated with reduced tumourigenesis is difficult to explain in light of the lack of effect of wheel running, and warrants further investigation.

Chronic inflammation increases the risk of colorectal cancer $^{(49)}$ possibly through oxidative damage from reactive oxygen and nitrogen species generated by neutrophils and macrophages in the inflamed tissue ${ }^{(50)}$. An enlarged spleen is symptomatic of chronic inflammation, as demonstrated by the development of colitis in IL-2-deficient mice ${ }^{(51)}$. Mehl et al. ${ }^{(17)}$ observed lower spleen size with exercise in male, but not in female, Min mice, and the authors suggested that reduced systemic inflammation in male mice may have been partially responsible for the reduction in tumour number. Our pooled data showing a positive correlation between spleen weight and tumour number support this hypothesis. In female C57BL/6 mice, Rogers et al. ${ }^{(38)}$ observed that wheel running reduced spleen weight and increased mucosal cytokine production and T-cell proliferation in Peyer's patch cells.

In summary, treadmill' running, but not wheel running, reduced slightly the number of larger $(\geq 2 \mathrm{~mm})$ intestinal tumours, and tended to reduce tumour multiplicity in the colon $(P=0.049)$ of Min mice. We have observed for the first time that treadmill exercise increases colonic butyrate in Min mice, and that there was an inverse correlation between butyrate and tumour multiplicity. Finally, we present novel observations of relationships between sleep duration and both tumour multiplicity and tumour burden. These findings offer novel avenues for exploration of mechanisms through which exercise (and other lifestyle interventions) may prevent intestinal tumourigenesis. 


\section{Acknowledgements}

The authors thank Adéle Kitching, Fiona Maclachlan and Emilie Guillaume for technical assistance. The present project was funded by the World Cancer Research Fund, project number 2001/38. L. B. and J. C. M. were involved in the study design, analysis and paper writing, and L. B. was involved in data collection. There are no conflicts of interest to declare.

\section{References}

1. Lee IM (2003) Physical activity and cancer prevention - data from epidemiologic studies. Med Sci Sports Exerc 35, $1823-1827$.

2. McTiernan A, Ulrich C, Slate S, et al. (1998) Physical activity and cancer etiology: associations and mechanisms. Cancer Causes Control 9, 487-509.

3. Stein CJ \& Colditz GA (2004) Modifiable risk factors for cancer. Br J Cancer 90, 299-303.

4. Slattery ML, Levin TR, Ma K, et al. (2003) Family history and colorectal cancer: predictors of risk. Cancer Causes Control 14, 879-887.

5. Colbert LH, Lanza E, Ballard-Barbash R, et al. (2002) Adenomatous polyp recurrence and physical activity in the Polyp Prevention Trial (United States). Cancer Causes Control 13, 445-453.

6. Wallace K, Baron JA, Karagas MR, et al. (2005) The association of physical activity and body mass index with the risk of large bowel polyps. Cancer Epidemiol Biomarkers Prev 14, 2082-2086.

7. WCRF/AICR (2007) Food, Nutrition, Physical Activity and the Prevention of Cancer: A Global Perspective. Washington, DC: American Institute for Cancer Research.

8. Basterfield L, Reul JMHM \& Mathers JC (2005) Impact of physical activity on intestinal cancer development in mice. J Nutr 135, 3002S-3008S.

9. Thorling EB, Jacobsen NO \& Overvad K (1993) Effect of exercise on intestinal tumour development in the male Fischer rat after exposure to azoxymethane. Eur J Cancer Prev 2, $77-82$.

10. Thorling EB, Jacobsen NO \& Overvad K (1994) The effect of treadmill exercise on azoxymethane-induced intestinal neoplasia in the male fischer rat on 2 different high-fat diets. Nutr Cancer 22, 31-41.

11. Klurfeld DM, Welch CB, Einhorn E, et al. (1988) Inhibition of colon-tumor promotion by caloric restriction or exercise in rats. FASEB $J \mathbf{2}, \mathrm{A} 433$.

12. Andrianopoulos G, Nelson RL, Bombeck CT, et al. (1987) The influence of physical activity in 1,2 dimethylhydrazine induced colon carcinogenesis in the rat. Anticancer Res 7, 849-852.

13. Reddy BS, Sugie S \& Lowenfels A (1988) Effect of voluntary exercise on azoxymethane-induced colon carcinogenesis in male F344 rats. Cancer Res 48, 7079-7081.

14. Moser AR, Pitot HC \& Dove WF (1990) A dominant mutation that predisposes to multiple intestinal neoplasia in the mouse. Science 247, 322-324.

15. Colbert LH, Davis JM, Essig DA, et al. (2000) Exercise and tumor development in a mouse predisposed to multiple intestinal adenomas. Med Sci Sports Exerc 32, 1704-1708.

16. Colbert LH, Mai V, Perkins SN, et al. (2003) Exercise and intestinal polyp development in APC(Min) mice. Med Sci Sports Exerc 35, 1662-1669.

17. Mehl KA, Davis JM, Clements JM, et al. (2005) Decreased intestinal polyp multiplicity is related to exercise mode and gender in Apc(Min/+) mice. J Appl Physiol 98, 2219-2225.
18. Colbert LH, Mai V, Tooze JA, et al. (2006) Negative energy balance induced by voluntary wheel running inhibits polyp development in APC(Min) mice. Carcinogenesis 27, $2103-2107$

19. Ju JH, Nolan B, Cheh M, et al. (2008) Voluntary exercise inhibits intestinal tumorigenesis in $\mathrm{Apc}(\mathrm{Min} /+)$ mice and azoxymethane/dextran sulfate sodium-treated mice. $B M C$ Cancer 8, 8.

20. Baltgalvis KA, Berger FG, Pena MMO, et al. (2009) The interaction of a high-fat diet and regular moderate intensity exercise on intestinal polyp development in ApcMin/+ mice. Cancer Prev Res 2, 641-649.

21. Jennings JR, Muldoon MF, Hall M, et al. (2007) Self-reported sleep quality is associated with the metabolic syndrome. Sleep 30, 219-223.

22. Vorona RD, Winn MP, Babineau TW, et al. (2005) Overweight and obese patients in a primary care population report less sleep than patients with a normal body mass index. Arch Intern Med 165, 25-30.

23. Blask DE (2009) Melatonin, sleep disturbance and cancer risk. Sleep Med Rev 13, 257-264.

24. Verkasalo PK, Lillberg K, Stevens RG, et al. (2005) Sleep duration and breast cancer: a prospective cohort study. Cancer Res 65, 9595-9600.

25. Wu AH, Wang RW, Koh WP, et al. (2008) Sleep duration, melatonin and breast cancer among Chinese women in Singapore. Carcinogenesis 29, 1244-1248.

26. Kakizaki M, Inoue K, Kuriyama S, et al. (2008) Sleep duration and the risk of prostate cancer: the Ohsaki Cohort Study. $\mathrm{Br} \mathrm{J}$ Cancer 99, 176-178.

27. Kakizaki M, Kuriyama S, Sone T, et al. (2008) Sleep duration and the risk of breast cancer: the Ohsaki Cohort Study. $\mathrm{Br} \mathrm{J}$ Cancer 99, 1502-1505.

28. Pinheiro SP, Schernhammer ES, Tworoger SS, et al. (2006) A prospective study on habitual duration of sleep and incidence of breast cancer in a large cohort of women. Cancer Res $\mathbf{6 6}$, 5521-5525.

29. Csordas A (1995) Toxicology of butyrate and short-chain fatty acids. In Role of Gut Bacteria in Human Toxicology and Pharmacology [MJ Hill, editor]. London: Taylor \& Francis Ltd.

30. Williams EA, Coxhead JM \& Mathers JC (2003) Anti-cancer effects of butyrate: use of micro-array technology to investigate mechanisms. Proc Nutr Soc 62, 107-115.

31. Su LK, Kinzler KW, Vogelstein B, et al. (1992) Multiple intestinal neoplasia caused by a mutation in the murine homolog of the APC gene. (Erratum appears in Science 1992 256, 1114). Science 256, 668-670.

32. Williamson SLH, Kartheuser A, Coaker J, et al. (1999) Intestinal tumorigenesis in the Apc1638N mouse treated with aspirin and resistant starch for up to 5 months. Carcinogenesis 20, 805-810.

33. Basterfield L, Lumley LK \& Mathers JC (2009) Wheel running in female C57BL/6J mice: impact of oestrus and dietary fat and effects on sleep and body mass. Int J Obes 33, 212-218.

34. Mathers JC \& Fotso Tagny JM (1994) Diurnal changes in largebowel metabolism - short-chain fatty-acids and transit-time in rats fed on wheat bran. Br J Nutr 71, 209-222.

35. McKay JA, Williams EA \& Mathers JC (2008) Gender-specific modulation of tumorigenesis by folic acid supply in the Apc $(+/$ Min) mouse during early neonatal life. Br J Nutr 99, 550-558.

36. Spindler SR (2005) Rapid and reversible induction of the longevity, anticancer and genomic effects of caloric restriction. Mech Ageing Dev 126, 960-966.

37. Mai V, Colbert LH, Berrigan D, et al. (2003) Calorie restriction and diet composition modulate spontaneous intestinal tumorigenesis in Apc(Min) mice through different mechanisms. Cancer Res 63, 1752-1755. 
38. Rogers CJ, Berrigan D, Zaharoff DA, et al. (2008) Energy restriction and exercise differentially enhance components of systemic and mucosal immunity in mice. $J$ Nutr $\mathbf{1 3 8}$, $115-122$.

39. Mehl KA, Davis JM, Berger FG, et al. (2005) Myofiber degeneration/regeneration is induced in the cachectic Apc(Min/+) mouse. J Appl Physiol 99, 2379-2387.

40. Baltgalvis KA, Berger FG, Pena MMO, et al. (2008) Interleukin-6 and cachexia in Apc(Min/+) mice. Am J Physiol Regul Integr Comp Physiol 294, R393-R401.

41. Wasan HS, Novelli M, Bee J, et al. (1997) Dietary fat influences on polyp phenotype in multiple intestinal neoplasia mice. Proc Natl Acad Sci U S A 94, 3308-3313.

42. WCRF/AICR (1997) Food, Nutrition and the Prevention of Cancer: A Global Perspective. Washington, DC: American Institute for Cancer Research.

43. Goodlad JS \& Mathers JC (1990) Large-bowel fermentation in rats given diets containing raw peas (Pisum sativum). Br J Nutr 64, 569-587.

44. Mai V, Colbert LH, Perkins SN, et al. (2007) Intestinal microbiota: a potential diet-responsive prevention target in $A p c^{\text {Min }}$ mice. Mol Carcinog 46, 42-48.
45. Matsumoto M, Inoue R, Tsukahara T, et al. (2008) Voluntary running exercise alters microbiota composition and increases n-butyrate concentration in the rat cecum. Biosci Biotechnol Biochem 72, 572-576.

46. Mathers JC \& Dawson LD (1991) Large bowel fermentation in rats eating processed potatoes. Br J Nutr 66, 313-329.

47. McElroy JA, Newcomb PA, Titus-Ernstoff L, et al. (2006) Duration of sleep and breast cancer risk in a large populationbased case-control study. J Sleep Res 15, 241-249.

48. Jenkins JB, Omori T, Guan Z, et al. (2006) Sleep is increased in mice with obesity induced by high-fat food. Physiol Behav 87, 255-262.

49. Coussens LM \& Werb Z (2002) Inflammation and cancer. Nature 420, 860-867.

50. Itzkowitz SH \& Yio X (2004) Inflammation and cancer IV. Colorectal cancer in inflammatory bowel disease: the role of inflammation. Am J Physiol Gastrointest Liver Physiol 287, G7-17.

51. Garrelds IM, Van Meeteren ME, Meijssen MAC, et al. (2002) Interleukin-2-deficient mice - effect on cytokines and inflammatory cells in chronic colonic disease. Dig Dis Sci 47, 503-510. 